\title{
Design and Implementation of Fractional-Order Microwave Integrator
}

\author{
Mridul GUPTA, Dharmendra Kumar UPADHYAY \\ Division of Electronics and Communication Engineering, NSIT, Sector-03, Dwarka, Delhi 110078, India \\ mri.gupta@gmail.com
}

Submitted October 23, 2018 / Accepted June 24, 2019

\begin{abstract}
A novel design of fractional-order microwave integrator using shunt connected open-stubs with transmission line sections in cascade is proposed. Design is obtained by optimizing the $L_{1-n o r m}$ based error function in $Z$-domain having not more than absolute magnitude error value of 0.01. Optimization is done using nature inspired cuckoo search algorithm. Superiority of the design in terms of magnitude error performance is identified by comparing it with the results obtained from some widely used benchmark optimization algorithms. The obtained design is implemented on a RT/Duroid 5880 substrate having 20 mil thickness, and results for the measured magnitude response are found to be in good agreement with ideal one over the frequency range of $1.0 \mathrm{GHz}$ to $6.8 \mathrm{GHz}$.
\end{abstract}

\section{Keywords}

Fractional-order, integrator, line elements, microwave, optimization

\section{Introduction}

An integrator is a physical device used to obtain time integral of a signal. Over past few decades, fractional calculus has attracted the research community to model a physical system with enhanced accuracy in comparison to an integer order model [1]. Fractional-order integrator (FOI) finds applicability in various fields of engineering and sciences [2]. Frequency response of an ideal FOI can be written as $H(\omega)=k /(\mathrm{j} \omega)^{\alpha}$, where $k$ is a scaling constant, $\omega$ is the angular frequency in radians per second and $\alpha$ is considered to be a fractional number, $\alpha \in(0,1)$.

For designing digital FOIs, two most widely used methods are direct and indirect discretization [3]. Based on these traditional methods, many research articles exist on the design of fractional-order infinite impulse response (IIR) integrators for digital signal processing applications [4-7]. In recent years, various nature inspired optimization algorithms like particle swarm optimization (PSO) [8], colliding bodies optimization [9], cuckoo search algorithm (CSA) [10] etc., had been studied extensively to obtain the optimal designs of FOIs. Here, highly accurate designs in terms of magnitude error responses were achieved using these algorithms. Root-mean-square and least-mean-square based error functions were formulated and minimized using various metaheuristic optimization algorithms to obtain the coefficients of a digital transfer function. It is noted that the similar methodology can be incorporated to design other Z-domain circuits.

Various design techniques were also identified in the literature to obtain integer-order IIR digital integrators. Simply by interpolating the rectangular, Simpson and trapezoidal rule integrators, IIR digital integrator of firstorder [11] and second-order [12] were obtained. Minmax based linear programing optimization was applied to obtain coefficients for the digital integrator [13]. Some numerical analysis techniques like Newton-Cotes integration, GaussLegendre integration and Richardson extrapolation were exploited for the design purpose [14-16]. Analysis was done by the authors to optimize pole-zero locations of existing IIR systems to obtain optimum magnitude and phase responses for digital integrators [17], [18]. Metaheuristic optimization algorithms like real-coded genetic algorithm (RCGA), artificial bee colony algorithm, differential evolution, PSO, bat algorithm, hybrid flower pollination algorithm etc. [19-22].

To estimate time integral of transmitted or received signals at $\mathrm{GHz}$ range, has always been a research issue in microwave engineering domain. Integrator circuits for microwave applications were obtained using the chain scattering parameters of transmission line elements in Z-domain. Using open-circuited stubs and transmission line sections, a trapezoidal-rule integrator was implemented by Hsue et al. [23]. Three different models for controlling the time constant of a microwave integrator were proposed to characterize its performance [24]. By applying the interpolation between Simpson and trapezoidal rule integrators in Z-domain, a microwave integrator was obtained and implemented in a microstrip format [25], [26]. First-order microwave integrator for interference suppression in RF circuits was implemented by Tsai [27], which exhibited an operating frequency range of $1.45 \mathrm{GHz}$ to $6 \mathrm{GHz}$.

It is identified that no work is done on the designing of circuits which can be used for obtaining fractional-inte- 
gral of a signal at microwave range. In this paper, a novel fractional-order microwave integrator (FOMI) design for $\alpha=0.5$ has been proposed using Z-domain chain scattering parameters of equal-length transmission line elements. Impedance values of the line elements are obtained through minimizing $L_{1-\text { norm }}$ based error function using CSA. The proposed design is implemented on a microstrip circuit using RT/Duroid 5880 substrate, which can be used for obtaining the time integral of a signal in fractional systems operating at microwave frequencies.

Remaining sections of this paper are organized as follows. Section 2 describes design methodology for the proposed FOMI. Configuration obtained through CSA is compared with the results from other optimization algorithms. Magnitude error performance of the proposed FOMI is compared with existing integer-order microwave integrators in Sec. 3. Microstrip implementation of the proposed FOMI configuration is done in Sec. 4. Finally, Section 5 concludes the work.

\section{Fractional-Order Microwave Integrator}

It is seen that an open circuited shunt-stub provides a zero at $z=-1(\omega=\pi)$ [23], which emulates the behavior of a low pass filter. Based on this concept, analysis is done in Z-domain to obtain the fractional-order $(\alpha=0.5) \mathrm{mi}$ crowave integrator design by cascading the transmission line sections with shunt connected open-stubs. Number and position of line elements are decided by approximating the magnitude response of cascaded configuration to that of an ideal FOI. Chain scattering parameters for equal-electricallength line elements are used for the design purpose. An open circuited shunt-stub and a transmission line section shown in Fig. 1 and 2 can be represented in Z-domain using the chain scattering matrices (T-matrices) given in (1) and (2), respectively [23].

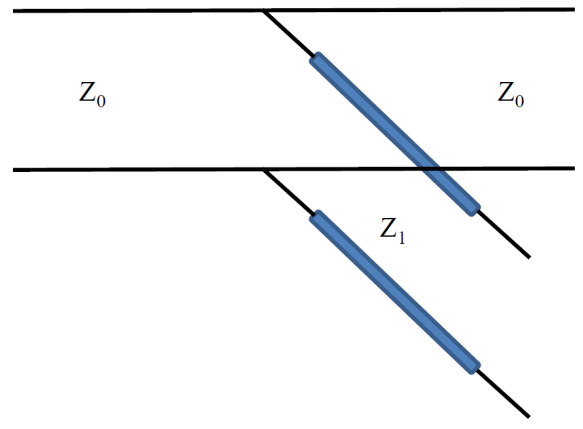

Fig. 1. Open circuited shunt stub.

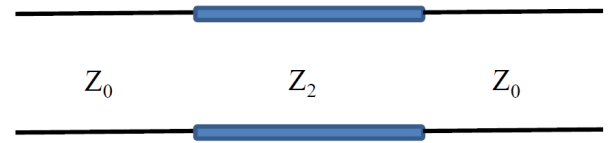

Fig. 2. Serial transmission line section.

$$
\begin{gathered}
\mathbf{T}_{\mathrm{OC}}=\frac{1}{\left(1+z^{-1}\right)}\left[\begin{array}{cc}
(1+r)+(1-r) z^{-1} & r-r z^{-1} \\
-r+r z^{-1} & (1-r)+(1+r) z^{-1}
\end{array}\right], \\
\mathbf{T}_{\mathrm{SL}}=\frac{1}{z^{-1 / 2}\left(1-K^{2}\right)}\left[\begin{array}{cc}
1-K^{2} z^{-1} & -\left(K-K z^{-1}\right) \\
K-K z^{-1} & -K^{2}+z^{-1}
\end{array}\right]
\end{gathered}
$$

where $z=\exp (\mathrm{j} \omega), r=Z_{0} /\left(2 Z_{1}\right)$ and the reflection coefficient $K=\left(Z_{2}-Z_{0}\right) /\left(Z_{2}+Z_{0}\right)$. Here, $Z_{1}$ represents the characteristic impedance of the open circuited shunt-stub, $Z_{2}$ is the characteristic impedance of a transmission line section and $Z_{0}$ is the termination impedance.

The overall transfer function for any cascaded network can be obtained by multiplying T-matrices of line elements. Chain scattering matrix parameter $T_{11 \text { overall }}(z)$, for the design consisting of $M$ transmission line sections and $N$ shunt connected open-stubs (both $M$ and $N$ are positive whole numbers) in cascade can be written as:

$$
T_{11 \text { overall }}(z)=\frac{\sum_{i=0}^{M+N} c_{i} z^{-i}}{z^{-M / 2}\left(1+z^{-1}\right)^{N} \prod_{m=1}^{M}\left(1-K_{m}^{2}\right)}
$$

where $z^{-M / 2}$ represents some delay corresponding to $M$ transmission line sections, $K_{m}$ 's are the reflection coefficients of the $M$-th transmission line section and $c_{i}$ 's are real numbers which depends on the characteristic impedances of all transmission line elements. By loading output of the considered network with matched termination, the overall transfer function can be defined as $G(z)=1 / T_{11 \text { overall }}(z)$. Neglecting delay $z^{-M / 2}$, the corresponding frequency response can be written as:

$$
G^{\prime}(\omega)=\frac{1}{T_{11 \text { overall }}^{\prime}(\omega)}=\frac{[1+\exp (-\mathrm{j} \omega)]^{N} \prod_{m=1}^{M}\left(1-K_{m}^{2}\right)}{\sum_{i=0}^{M+N} c_{i} \exp (-i \mathrm{j} \omega)} .
$$

To obtain the FOMI design, $G^{\prime}(\omega)$ is approximated to an ideal FOI $(\alpha=0.5)$ by calculating the absolute magnitude error $(A M E)$ between the two, using a $L_{1-\text { norm }}$ error function given by:

$$
\|E(\omega)\|=\sum_{\omega}\left\|G^{\prime}(\omega)|-| H(\omega)\right\|
$$

where \| $\|$ denotes the norm of the function. $L_{1-\text { norm }}$ based error function is chosen because it overcomes the shortcoming of having ripples in the frequency response over a wideband and high overshoots at discontinuous points, as compared to other approximation methods such as $L_{2}$ and $L_{\propto-\text { norm }}$ [21]. Global optimal solutions can be obtained using the $L_{1-\text { norm }}$ technique in conjunction with CSA. Also, it provides higher design flexibility and better absolute magnitude error performance [28]. Error function defined in (5) is minimized through the optimization process using CSA [29], to achieve an optimum design for the FOMI. In 
this algorithm, every egg laid by the cuckoo bird is treated as a possible solution, viz. characteristic impedance values of the line elements.

\subsection{Cuckoo Search Algorithm}

The cuckoo search algorithm, formulated by Yang and Deb [29] is based on the obligate brood parasitic behavior of cuckoo species. CSA, in combination with Lévy Flight behavior of some birds and fruit flies emanated as one of the modern metaheuristic algorithm which provides more robust and precise solution to a problem. As per its behavior, cuckoo bird lays egg in a nest where the host bird has recently laid eggs. In the event, the host bird discovers about foreign egg in its nest, it may either destroy the alien egg or abandon the nest and look for a new location. For the survival, species of cuckoo birds have evolved in such a way that eggs laid mimic in color and pattern of the eggs of a few host species. This reduces the probability of their eggs being abandoned which in turn increases their reproductivity.

Three idealized rules govern CSA viz., (i) Each cuckoo bird lays one egg at a time which is randomly placed among host bird's nests; (ii) The nest with the high quality eggs would carry over to the next generation; (iii) Number of host nests is fixed and the egg laid by cuckoo is discovered by the host bird with the probability $\mathrm{P}_{\mathrm{a}} \in[0,1]$.

In CSA, each cuckoo egg in the host's nest symbolizes a potential solution of the defined problem which is characterized by its fitness value. Thus, to generate a new solution of high fitness value, the concept of random walk performed by Lévy flights is applied. Steps taken by birds are random and depend on current location and transition probability to the next location. Mathematically, the new location, i.e. nest, with high fitness value is obtained from Lévy distribution with an infinite variance and mean as:

$$
a_{k+1}=a_{k}+\gamma_{0} \otimes \operatorname{Lévy}(\lambda)
$$

where $\gamma_{0}$ is the step size related to the problem specified and determines distance of the random walk, $\otimes$ represents entry wise multiplication and $\lambda$ is a Lévy flight parameter. The flow chart for the CSA based FOMI design method is explained in Fig. 3.

\subsection{Proposed FOMI Configuration}

For practical realization of microstrip circuits, lower and upper bounds for characteristic impedances of transmission line elements have been chosen as $10 \Omega \leq Z \leq 150 \Omega$. Termination impedance $Z_{\text {Term }}$ at both input and output ports is considered as $50 \Omega$. Optimization process is applied to obtain the FOMI configuration, by changing the position and number of both shunt connected open-stubs and transmission line sections. Numerous simulations are carried out by running the optimization algorithm to minimize the error function defined in (5) with acceptable $A M E$ value over the wideband. Figure 4 shows the configuration

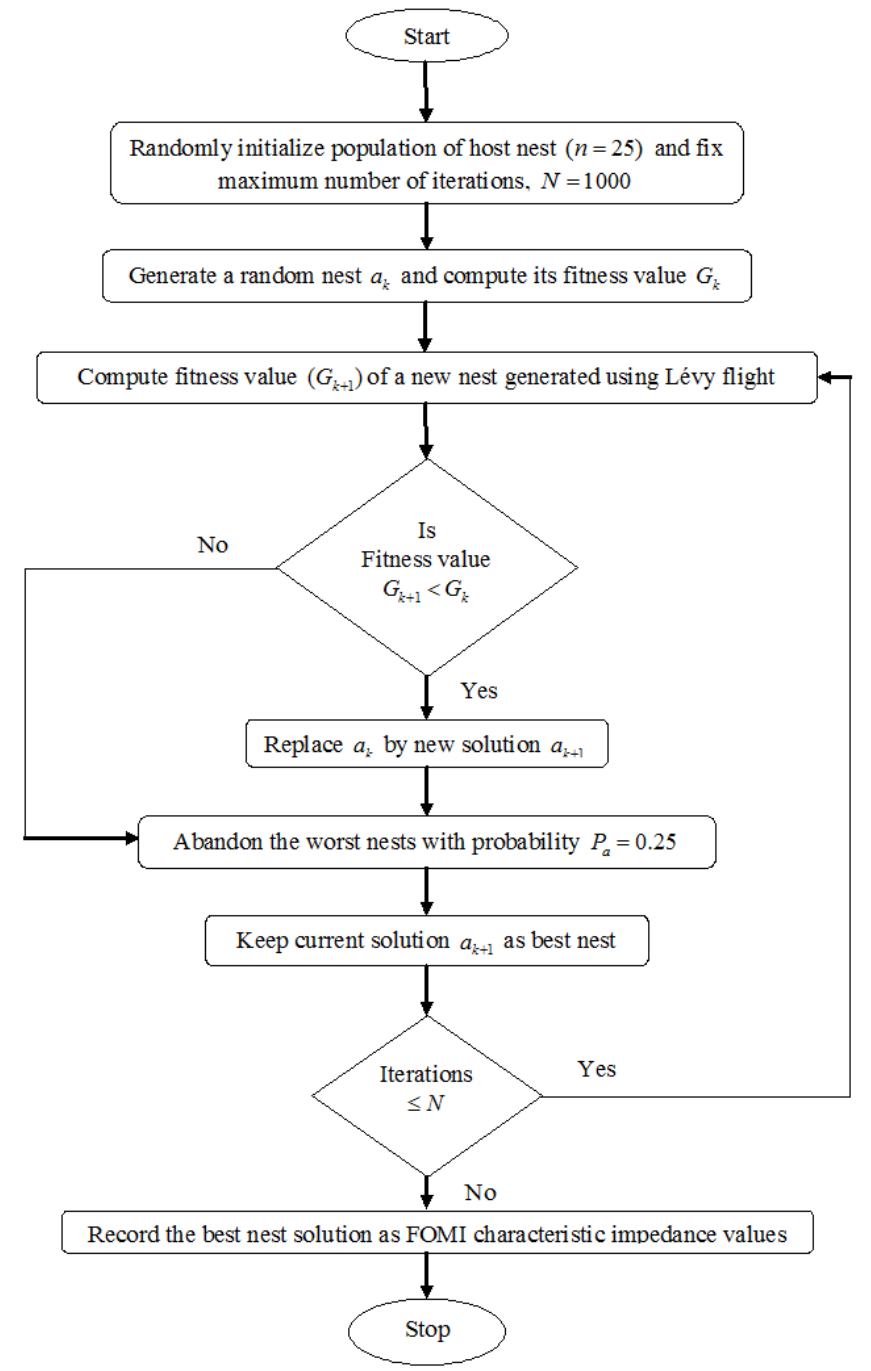

Fig. 3. Flow chart for the CSA based FOMI design method.

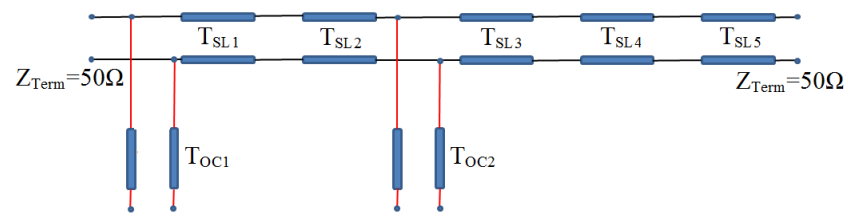

Fig. 4. Configuration of the proposed FOMI.

obtained for the proposed FOMI, with maximum $A M E$ value of 0.01 over the frequency range $0.1 \pi \leq \omega \leq 0.75 \pi$. Configuration consists of two shunt connected open-stubs $\left(\mathrm{T}_{\mathrm{OC} 1}\right.$ and $\left.\mathrm{T}_{\mathrm{OC} 2}\right)$ with five transmission line sections $\left(\mathrm{T}_{\mathrm{SL} 1}\right.$, $\mathrm{T}_{\mathrm{SL} 2}, \mathrm{~T}_{\mathrm{SL} 3}, \mathrm{~T}_{\mathrm{SL} 4}$, and $\mathrm{T}_{\mathrm{SL} 5}$ ) in cascade having characteristic impedance values from left to right as $\{135,56.4481$, $10.1382,138,10,64.2104,46.4273\} \Omega$.

Superiority of the design from CSA is verified by comparing with the results obtained from some widely used benchmark evolutionary optimization algorithms in the literature, namely, GSA [30], PSO [31] and RCGA [32] for the same configuration. All algorithms are made to run for 1000 iterations and corresponding control parameters are mentioned in Tab. 1 . 


\begin{tabular}{lll}
\hline Algorithm & Control Parameter & Value \\
\hline GSA & Gravitation constant & 100 \\
& Coefficient of decrease & 20 \\
& Number of agents & 50 \\
\hline PSO & Inertia weight & 1 \\
& Inertia damping ratio & 0.99 \\
& Personal learning coefficient & 1.5 \\
& Global learning coefficient & 2 \\
& Swarm size & 100 \\
\hline RCGA & Population size & 100 \\
& Crossover percentage & 0.7 \\
& Mutation percentage & 0.3 \\
& Mutation rate & 0.1 \\
& Selection type: Roulette wheel & \\
\hline
\end{tabular}

Tab. 1. Control parameters for different optimization algorithms.

\begin{tabular}{lllll}
\hline \multirow{2}{*}{$\begin{array}{l}\text { Line } \\
\text { Element }\end{array}$} & \multicolumn{4}{c}{ Impedances $(\boldsymbol{\Omega})$} \\
\cline { 2 - 5 } & CSA & GSA & PSO & RCGA \\
\hline $\mathrm{T}_{\mathrm{OC} 1}$ & 135 & 139.5694 & 128.5752 & 139.9891 \\
$\mathrm{~T}_{\mathrm{SL} 1}$ & 56.4481 & 53.7007 & 49.9875 & 36.1933 \\
$\mathrm{~T}_{\mathrm{SL} 2}$ & 10.1382 & 10.7479 & 17.9110 & 10 \\
$\mathrm{~T}_{\mathrm{OC} 2}$ & 138 & 131.2516 & 41.5766 & 29.1531 \\
$\mathrm{~T}_{\mathrm{SL} 3}$ & 10 & 10.0981 & 10 & 16.2060 \\
$\mathrm{~T}_{\mathrm{SL} 4}$ & 64.2104 & 68.8665 & 73.0595 & 88.1789 \\
$\mathrm{~T}_{\mathrm{SL} 5}$ & 46.4273 & 49.9386 & 67.5758 & 40.3155 \\
\hline
\end{tabular}

Tab. 2. Characteristic impedance values from different optimization algorithms.

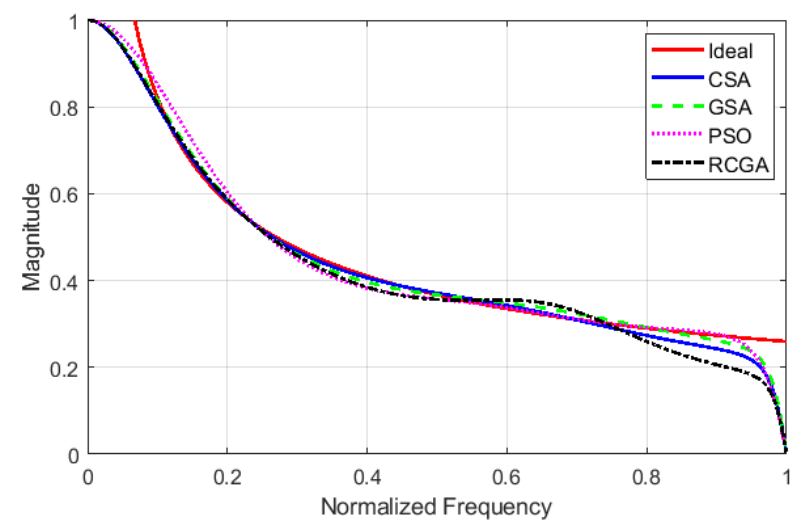

Fig. 5. Magnitude responses of the designs obtained through different optimization algorithms.

Values of characteristic impedances for the configuration with best error performance obtained by running each algorithm are listed in Tab. 2. The corresponding magnitude responses of the designs obtained through these algorithms are shown is Fig. 5.

Figure 6 shows the convergence curve for cumulative error values of CSA, PSO, RCGA and GSA over wide band with increase in the number of iterations. Although, GSA converges at a very early stage but it is seen to have higher error value as compared to other optimization algorithms. RCGA shows the slowest rate of convergence with improved error performance compared to GSA. PSO and CSA converge to the minimum error value and give the most optimum results for FOMI design.

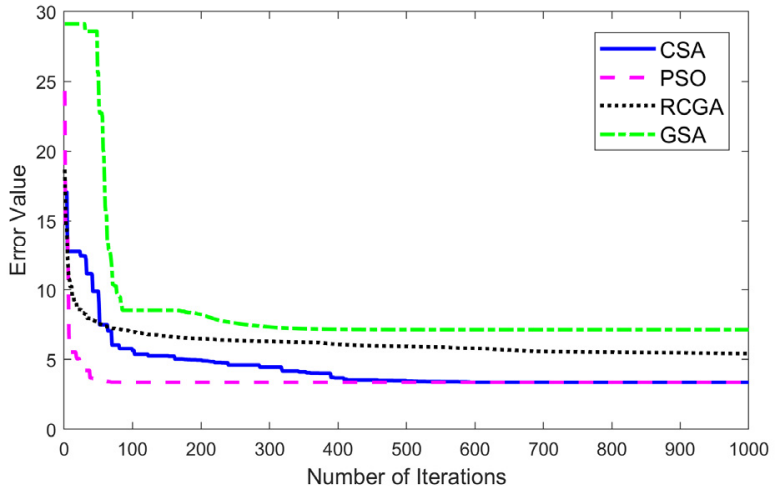

Fig. 6. Convergence curve of CSA, PSO, RCGA and GSA for obtaining FOMI designs.

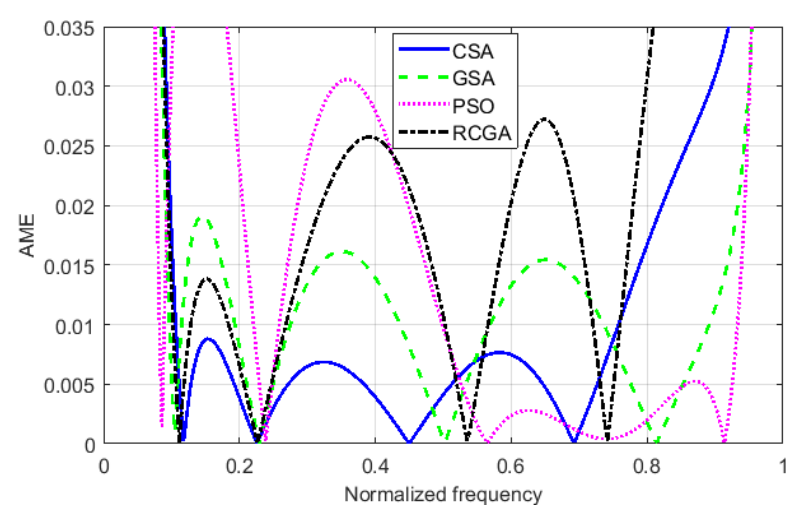

Fig. 7. AME comparison of the designs obtained through different optimization algorithms.

Accuracy of all the obtained designs is now tested through the $A M E$ comparison plot as given in Fig. 7. Among all four optimization algorithms used, the design obtained through PSO shows poor error performance at lower band but is having very low $A M E$ value at the upper operating frequency range. CSA is found to give highly accurate FOMI design with $A M E$ value of 0.01 over the maximum operating bandwidth $(0.1 \pi \leq \omega \leq 0.75 \pi)$ as compared to the designs by GSA, PSO and RCGA.

The proposed methodology is a generalized transmission line based approach and can be used for obtaining other microwave circuits using the metaheuristic optimization techniques.

Furthermore, magnitude error performance analysis of various designs is also done based on the following defined error parameters (in $\mathrm{dB})$ :

i. Maximum Absolute Magnitude Error $\left(\operatorname{Max}_{A M E}\right)$

$$
\operatorname{Max}_{A M E}=20 \log _{10}(\max |E(\omega)|)
$$

ii. Mean Absolute Magnitude Error $\left(\operatorname{Mean}_{A M E}\right)$

$$
\operatorname{Mean}_{A M E}=20 \log _{10}\left(\frac{\sum_{\omega}|E(\omega)|}{Q}\right)
$$

where $Q=400$ is the number of considered sample frequency points. 


\begin{tabular}{ccc}
\hline Optimization & \multicolumn{2}{c}{ Error Parameter } \\
\cline { 2 - 3 } Algorithm & $\operatorname{Max}_{A M E}(\mathrm{~dB})$ & Mean $_{A M E}(\mathrm{~dB})$ \\
\hline RCGA & -31.30 & -36.46 \\
PSO & -25.30 & -35.37 \\
GSA & -34.36 & -39.24 \\
CSA & $-\mathbf{3 5 . 1 7}$ & $\mathbf{- 4 6 . 2 5}$ \\
\hline
\end{tabular}

Tab. 3. Error parameter comparison for different FOMI designs.

For FOMI designs obtained from different optimization algorithms, values of the above defined parameters are calculated and listed in Tab. 3. It is clearly seen that the design obtained from CSA have the least values of maximum and mean $A M E$ over the specified band as compared to other optimization algorithm designs.

\section{Comparison}

Literature survey yields that no work has been done on the design of fractional-order microwave integrator circuits. Although, it is seen that many researchers are constantly working on design and implementation of integer-order microwave integrators for more than a decade. First-order microwave integrator designs were proposed using chain-scattering parameters of equal-length transmission line elements [23-27]. Accuracy of these integer-order designs in terms of magnitude error performance is compared with that of the proposed FOMI. Figure 8 clearly shows that the proposed FOMI design has comparatively much superior $A M E$ response over the existing integer-order

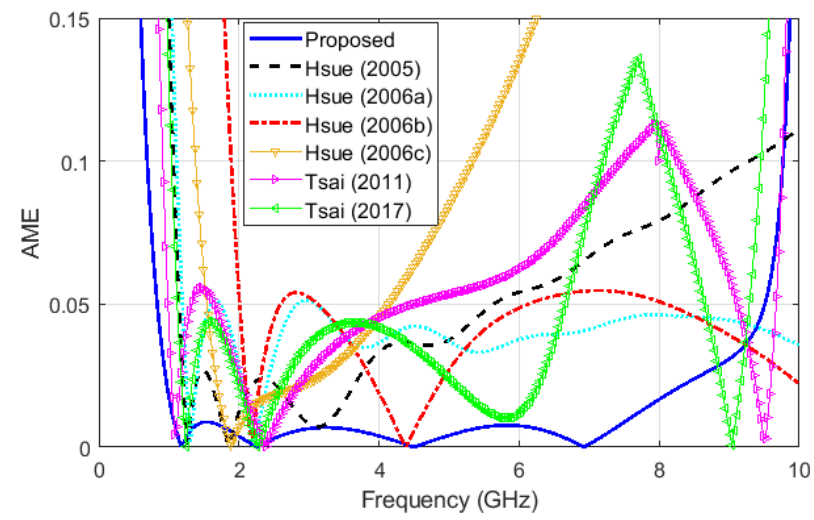

Fig. 8. AME comparison of proposed FOMI with existing integer-order microwave integrators.

\begin{tabular}{ccc}
\hline \multirow{2}{*}{ Integrator Design } & \multicolumn{2}{c}{ Error Parameter $(0.1 \pi \leq \omega \leq 0.75 \pi)$} \\
\cline { 2 - 3 } & $\operatorname{Max}_{A M E}(\mathrm{~dB})$ & $\operatorname{Mean}_{A M E}(\mathrm{~dB})$ \\
\hline Hsue (2005) & -16.28 & -28.61 \\
Hsue (2006a) & -14.39 & -27.95 \\
Hsue (2006b) & 0.41 & -20.14 \\
Hsue (2006c) & -8.97 & -20.61 \\
Tsai (2011) & -19.93 & -26.24 \\
Tsai (2017) & -17.96 & -28.65 \\
Proposed FOMI & $-\mathbf{3 5 . 1 7}$ & $\mathbf{- 4 6 . 2 5}$ \\
\hline
\end{tabular}

Tab. 4. Error parameter comparison of proposed FOMI with existing integer-order microwave integrators. microwave integrators. For the frequency range of $1 \mathrm{GHz}$ to $7.5 \mathrm{GHz}$, the proposed FOMI configuration gives highly accurate magnitude response with not more than $A M E$ value of 0.01 . Furthermore, values of the error parameters defined in (7) and (8) are calculated for the proposed normalized frequency range and are listed in Tab. 4. The best values of $\operatorname{Max}_{A M E}$ and $M_{e a n_{A M E}}$ over the specified band are found for the proposed FOMI design as compared to the existing integer-order microwave integrators.

\section{Implementation of FOMI}

The obtained FOMI design is fabricated on RT/Duroid 5880 substrate of 20 mil thickness having relative permittivity $\varepsilon_{\mathrm{r}}=2.2$ with loss tangent value of 0.0009 . The equal-electrical length of all transmission line elements is taken as $90^{\circ}$. We have considered the physical length of all line elements as $l=\lambda_{0} / 4$ in which $\lambda_{0}$ denotes the wavelength at normalizing frequency of $10 \mathrm{GHz}$. Physical dimensions of the proposed FOMI configuration are listed in Tab. 5. The total length of the fabricated FOMI, excluding that of the termination impedances is $28 \mathrm{~mm}$.

\begin{tabular}{cccccccc}
\hline $\begin{array}{c}\text { Line } \\
\text { Element }\end{array}$ & $\mathrm{T}_{\mathrm{OC} 1}$ & $\mathrm{~T}_{\mathrm{SL} 1}$ & $\mathrm{~T}_{\mathrm{SL} 2}$ & $\mathrm{~T}_{\mathrm{OC} 2}$ & $\mathrm{~T}_{\mathrm{SL} 3}$ & $\mathrm{~T}_{\mathrm{SL} 4}$ & $\mathrm{~T}_{\mathrm{SL} 5}$ \\
\hline $\begin{array}{c}\text { Width } \\
\text { (mm) }\end{array}$ & 0.17 & 1.26 & 11.48 & 0.16 & 11.66 & 1.01 & 1.71 \\
\hline
\end{tabular}

Tab. 5. Physical dimensions of the proposed FOMI.

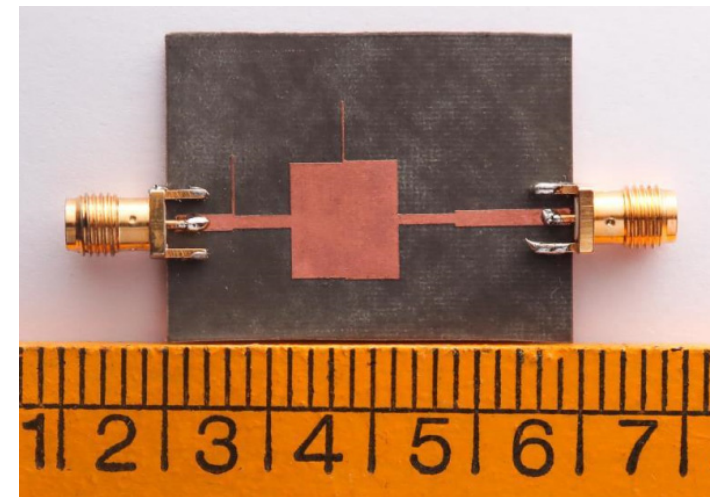

(a)

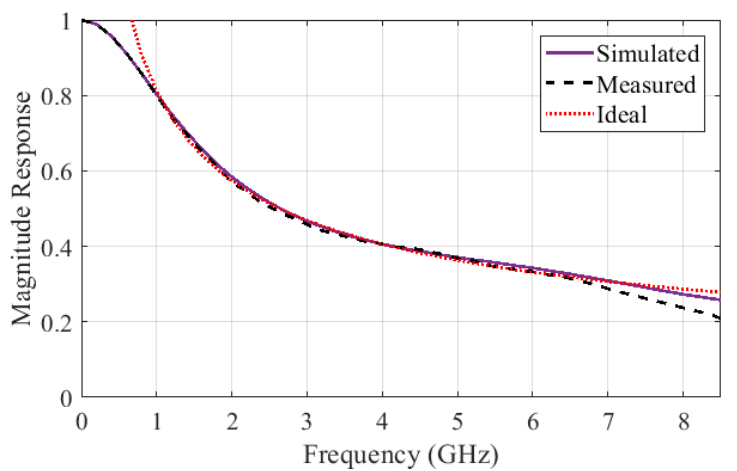

(b)

Fig. 9. Fractional-order microwave integrator: (a) Photograph of the fabricated FOMI. (b) Magnitude response comparison for the simulated, measured and ideal $S_{21}(f)$. 
Figures 9(a) and 9(b) respectively show photograph of the fabricated FOMI and its magnitude response comparison for the measured transmission coefficient $S_{21}(f)$ with simulated and the ideal ones. It is seen that the measured results for the fabricated FOMI validated the simulated results up to an operating frequency of $6.8 \mathrm{GHz}$ for the proposed design. Variation between the two arises mainly because of the fabrication tolerances and the mismatch occurring due to the connector joints, which could not be taken into account during the designing and simulation of the FOMI. Furthermore, measured results closely follow the ideal one over the frequency range of $1.0 \mathrm{GHz} \leq f \leq 6.8 \mathrm{GHz}$.

\section{Conclusion}

A fractional-order microwave integrator is proposed using equal-electrical-length transmission line elements. The obtained FOMI configuration consists of two shunt connected open-stubs with five transmission line sections in cascade. Highly accurate magnitude response is achieved through the optimization process. The proposed FOMI design outperforms the existing integer-order microwave integrators in terms of magnitude error performance with maximum and mean AME values of $-35.17 \mathrm{~dB}$ and $-46.25 \mathrm{~dB}$ respectively over the specified frequency range. The design is implemented on a microstrip circuit and measured results for magnitude response are found to be in good agreement to that of the ideal one over the operating frequency range $1.0 \mathrm{GHz} \leq f \leq 6.8 \mathrm{GHz}$. The proposed FOMI is the first of its kind and can be used to obtain the fractional-order time integral of signals for microwave applications. It can be placed in a radio receiver to improve signal-to-noise ratio over the communication link. Also, it is well suited for various other microwave applications such as Bluetooth, Wi-Fi, WIMAX, etc.

\section{References}

[1] WeSt, B. J., BOLOGNA, M., GRIGOLINI, P. Physics of Fractal Operators. New York: Springer, 2003. DOI: 10.1007/978-0-38721746-8

[2] DEBNATH, L. Recent applications of fractional calculus to science and engineering. International Journal of Mathematics and Mathematical Sciences, 2003, vol. 54, p. 3413-3442. DOI: $10.1155 / \mathrm{S} 0161171203301486$

[3] CHEN, Y. Q., MOORE, K. L. Discretization schemes for fractional-order differentiators and integrators. IEEE Transactions on Circuits and Systems I: Fundamental Theory and Applications, 2003, vol. 49, no. 3, p. 363-367. DOI: $10.1109 / 81.989172$

[4] BARBoSA, R. S., MACHADO, J. A. T., SILVA, M. F. Time domain design of fractional differintegrators using least-squares. Signal Processing, 2006, vol. 86, no. 10, p. 2567-2581. DOI: 10.1016/j.sigpro.2006.02.005

[5] TSENG, C. C. Design of FIR and IIR fractional order Simpson digital integrators. Signal Processing, 2007, vol. 87, no. 5, p. 1045-1057. DOI: 10.1016/j.sigpro.2006.09.006
[6] KRISHNA, B. T. Studies of fractional order differentiators and integrators: A survey. Signal Processing, 2011, vol. 91, no. 3, p. 386-426. DOI: 10.1016/j.sigpro.2010.06.022

[7] ROMERO, M., DE MADRID, A. P., MANOSO, C., et al. IIR approximations to the fractional differentiator/integrator using Chebyshev polynomials theory. ISA Transactions, 2013, vol. 52, no. 4 , p. 461-468. DOI: $10.1016 /$ j.isatra.2013.02.002

[8] YADAV, R., GUPTA, M. New improved fractional order integrators using PSO optimization. International Journal of Electronics, 2015, vol. 102, no. 3, p. 490-499. DOI: 10.1080/00207217.2014.901424

[9] MAHATA, S., SAHA, S. K., KAR, R., MANDAL, D. Optimal design of wideband infinite impulse response fractional order digital integrators using colliding bodies optimisation algorithm. IET Signal Processing, 2016, vol. 10, no. 9, p. 1135-1156. DOI: 10.1049/iet-spr.2016.0298

[10] MAHATA, S., SAHA, S. K., KAR, R., MANDAL, D. Infinite impulse response approximations to the non-integer order integrator using Cuckoo Search Algorithm. In: Saeed, K., Homenda, W., Chaki, R. (eds.) Computer Information Systems and Industrial Management CISIM 2017. 2017, p. 548-556. DOI: 10.1007/978-3-319-59105-6 47

[11] LE BIHAN, J. Novel class of digital integrators and differentiators. Electronics Letters, 1993, vol. 29, no. 11, p. 971-973. DOI: 10.1049/el:19930647

[12] AL-ALAOUI, M. A. A class of second order integrators and lowpass differentiators. IEEE Transactions on Circuits and Systems I: Fundamental Theory and Applications, 1995, vol. 42, no. 4, p. 220-223. DOI: $10.1109 / 81.382477$

[13] PAPAMARKOS, N., CHAMZAS, C. A new approach for the design of digital integrators. IEEE Transactions on Circuits and Systems I: Fundamental Theory and Applications, 1996, vol. 43, no. 9 , p. 785-791. DOI: $10.1109 / 81.536749$

[14] NGO, N. Q. A new approach for the design of wideband digital integrator and differentiator. IEEE Transactions on Circuits and Systems II: Express Briefs, 2006, vol. 53, no. 9, p. 936-940. DOI: 10.1109/TCSII.2006.881806

[15] TSENG, C.C., LEE, S. L. Digital IIR integrator design using Richardson extrapolation and fractional delay. IEEE Transactions on Circuits and Systems I: Regular Papers, 2008, vol. 55, no. 8, p. 2300-2309. DOI: 10.1109/TCSI.2008.920099

[16] AL-ALAOUI, M. A. Class of digital integrators and differentiators. IET Signal Processing, 2011, vol. 5, no. 2, p. 251-260. DOI: 10.1049/iet-spr.2010.0107

[17] UPADHYAY, D. K., SINGH, R. K. Recursive wideband digital differentiator and integrator. Electronics Letters, 2011, vol. 47, no. 11, p. 647-648. DOI: 10.1049/el.2011.0420

[18] UPADHYAY, D. K. Class of recursive wideband digital differentiators and integrators. Radio Engineering, 2012, vol. 21, no. 3, p. 904-910. ISSN: 1210-2512

[19] KARABOGA, N. A new design method based on artificial bee colony algorithm for digital IIR filters. Journal of the Franklin Institute, 2009, vol. 346, no. 4, p. 328-348. DOI: 10.1016/j.jfranklin.2008.11.003

[20] JALLOUL, M. K., AL-ALAOUI, M. A. Design of recursive digital integrators and differentiators using particle swarm optimization. International Journal of Circuit Theory and Applications, 2016, vol. 44, no. 5, p. 948-967. DOI: 10.1002/cta.2115

[21] AGGARWAL, A., RAWAT, T. K., UPADHYAY, D. K. Optimal design of L1-norm based IIR digital differentiators and integrators using the bat algorithm. IET Signal Processing, 2017, vol. 11, no. 1 , p. 26-35. DOI: 10.1049/iet-spr.2016.0010 
[22] MAHATA, S., SAHA, S. K., KAR, R., MANDAL, D. Optimal design of wideband digital integrators and differentiators using hybrid flower pollination algorithm. Soft Computing, 2018, vol. 22 , no. 11 , p. 3757-3783. DOI: $10.1007 / \mathrm{s} 00500-017-2595-6$

[23] HSUE, C. W., TSAI, L. C., KAN, S. T. Implementation of a trapezoidal rule microwave integrator. Microwave and Optical Technology Letters, 2005, vol. 48, no. 4, p. 822-825. DOI: 10.1002/mop. 21485

[24] HSUE, C. W., TSAI, L. C., TSAI, Y. H. Time constant control of microwave integrators using transmission lines. IEEE Transactions on Microwave Theory and Techniques, 2006, vol. 54, no. 3, p. 1043-1047. DOI: 10.1109/TMTT.2006.869722

[25] TSAI, L. C., FANG, H. S. Design and implementation of secondorder microwave integrators. Microwave and Optical Technology Letters, 2011, vol. 53, no. 9, p. 1983-1986. DOI: $10.1002 /$ mop. 26210

[26] GUPTA, M., UPADHYAY, D. K. Comments on: Design and implementation of second-order microwave integrators. Microwave and Optical Technology Letters, 2018, vol. 60, no. 2, p. 526-528. DOI: 10.1002/mop.30996

[27] TSAI, L. C. Application of microwave integrators for interference suppression. Progress In Electromagnetics Research C, 2017, vol. 72, p. 123-132. DOI: 10.2528/PIERC16112505

[28] AGGARWAL, A., KUMAR, M., RAWAT, T. K., et al. Optimal design of 2-D FIR digital differentiator using L1-norm based cuckoo-search algorithm. Multidimensional Systems and Signal Processing, 2017, vol. 28, no. 4, p. 1569-1567. DOI: 10.1007/s11045-016-0433-0

[29] YANG, X. S., DEB, S. Cuckoo search via Lévy flights. In Proceeding of World Congress on Nature and Biologically Inspired Computing. Coimbatore (India), 2009, p. 210-214. DOI: 10.1109/NABIC.2009.5393690

[30] RASHEDI, E., NEZAMABADI-POUR, H., SARYAZDI, S. GSA: A gravitational search algorithm. Information Sciences, 2009, vol. 179, no. 13, p. 2232-2248. DOI: 10.1016/j.ins.2009.03.004

[31] MARINI, F., WALCZAK, B. Particle swarm optimization (PSO): A tutorial. Chemometrics and Intelligent Laboratory Systems,
2015, vol. 149, Part B, p. 153-165. DOI: 10.1016/j.chemolab.2015.08.020

[32] CHUANG, Y. C., CHEN, C. T., HWANG, C. A simple and efficient real-coded genetic algorithm for constrained optimization. Applied Soft Computing, 2015, vol. 38, p. 87-105. DOI: 10.1016/j.asoc.2015.09.036

\section{About the Authors ...}

Mridul GUPTA passed his B. Tech in Electronics and Communication Engineering in the year 2010 from Uttarakhand Technical University, Uttarakhand, India. He received his M-Tech degree from Motilal Nehru National Institute of Technology, Allahabad, India in the year 2015. $\mathrm{He}$ is currently pursuing Ph.D. in the area of optimal design and implementation of digital and microwave filters from Netaji Subhas Institute of Technology, University of Delhi, India. His research areas include optimization techniques, signal processing, fractional-order systems and microwave circuits.

Dharmendra Kumar UPADHYAY received his M-Tech degree from Aligarh Muslim University, U.P., India. He did Ph.D. from Uttarakhand Technical University, Uttarakhand, India. Presently, he is attached with Netaji Subhas Institute of Technology, Delhi University, Delhi, India, as Professor in the Division of Electronics and Communication Engineering. He is having more than 20 years of teaching and research experience. His research interests include digital signal processing, microwave and array antenna design and filter optimization via nature-inspired computational techniques. He has published more than 50 research papers in reputed international journals and conferences. 